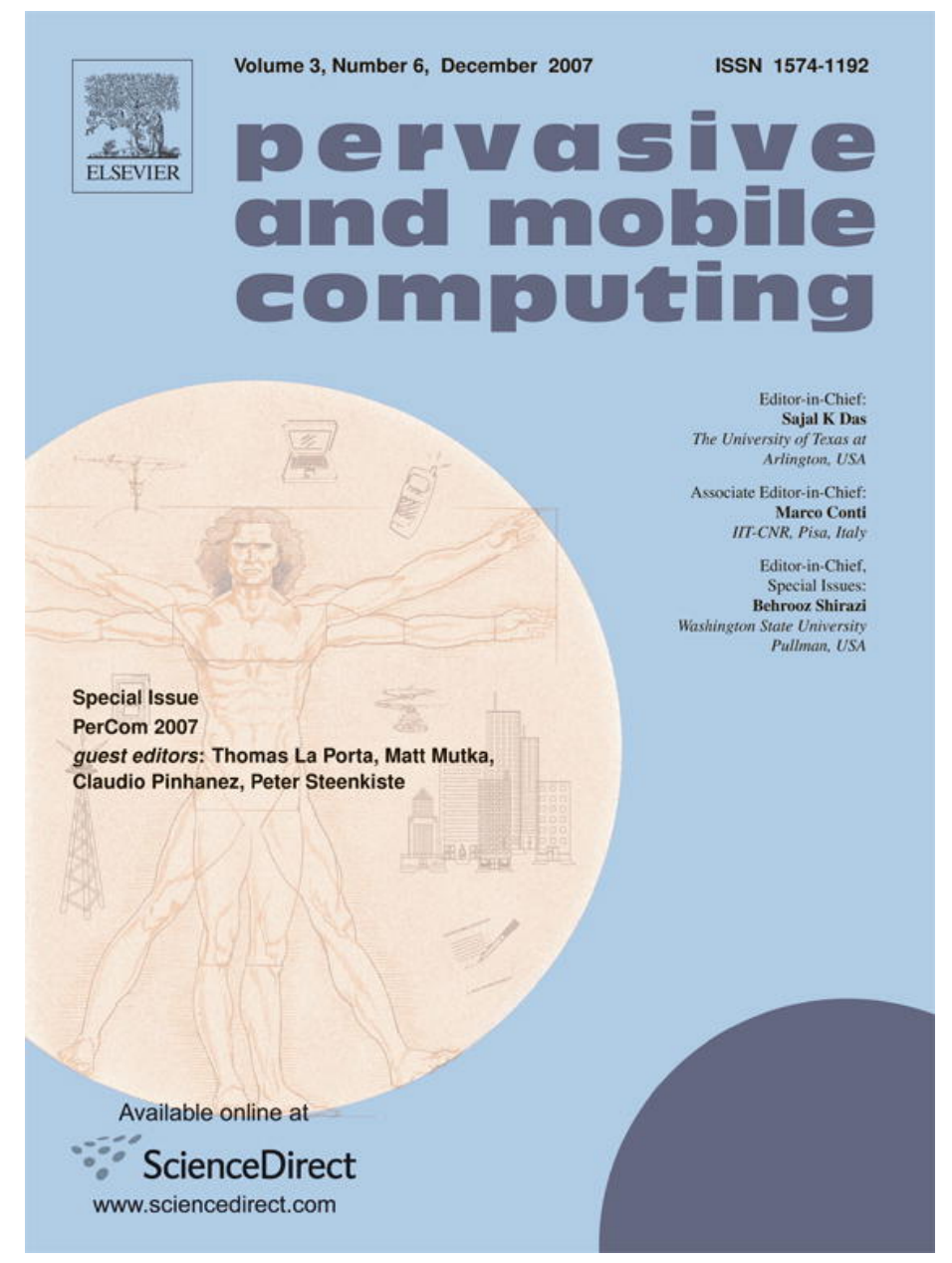

This article was published in an Elsevier journal. The attached copy

is furnished to the author for non-commercial research and education use, including for instruction at the author's institution, sharing with colleagues and providing to institution administration.

Other uses, including reproduction and distribution, or selling or licensing copies, or posting to personal, institutional or third party websites are prohibited.

In most cases authors are permitted to post their version of the article (e.g. in Word or Tex form) to their personal website or institutional repository. Authors requiring further information regarding Elsevier's archiving and manuscript policies are encouraged to visit: 


\title{
GSM indoor localization
}

\author{
Alex Varshavsky ${ }^{\mathrm{a}, *}$, Eyal de Lara ${ }^{\mathrm{a}}$, Jeffrey Hightower ${ }^{\mathrm{c}}$, \\ Anthony LaMarca ${ }^{\mathrm{c}}$, Veljo Otsason ${ }^{\mathrm{b}}$ \\ ${ }^{a}$ Computer Science Department, University of Toronto, 40 St. George Street, Toronto, Ontario M5S2E4, Canada \\ ${ }^{\mathrm{b}}$ Computer Science Department, University of Tartu, Ulikooli 18, 50090 Tartu, Estonia \\ ${ }^{\mathrm{c}}$ Intel Research Seattle, 1100 NE 45th St., Seattle, WA 98105, USA
}

Received 31 March 2007; received in revised form 22 June 2007; accepted 9 July 2007

Available online 31 July 2007

\begin{abstract}
Accurate indoor localization has long been an objective of the ubiquitous computing research community, and numerous indoor localization solutions based on 802.11, Bluetooth, ultrasound and infrared technologies have been proposed. This paper presents the first accurate GSM indoor localization system that achieves median within floor accuracy of $4 \mathrm{~m}$ in large buildings and is able to identify the floor correctly in up to $60 \%$ of the cases and is within 2 floors in up to $98 \%$ of the cases in tall multi-floor buildings. We report evaluation results of two case studies conducted over a course of several years, with data collected from 6 buildings in 3 cities across North America. The key idea that makes accurate GSM-based indoor localization possible is the use of wide signalstrength fingerprints. In addition to the 6-strongest cells traditionally used in the GSM standard, the wide fingerprint includes readings from additional cells that are strong enough to be detected, but are too weak to be used for efficient communication. We further show that selecting a subset of highly relevant channels for fingerprinting matching out of all available channels, further improves the localization accuracy.
\end{abstract}

(c) 2007 Elsevier B.V. All rights reserved.

Keywords: Localization; Fingerprinting; GSM; Ubicomp; Location

\footnotetext{
* Corresponding author. Tel.: +1 4169460241 .

E-mail addresses: walex@cs.toronto.edu (A. Varshavsky), delara@cs.toronto.edu (E. de Lara), jeffrey.r.hightower@intel.com (J. Hightower), anthony.lamarca@intel.com (A. LaMarca), veljo.otsason@utoronto.ca (V. Otsason).
}

1574-1192/\$ - see front matter (C) 2007 Elsevier B.V. All rights reserved. doi:10.1016/j.pmcj.2007.07.004 


\section{Introduction}

The accurate localization of objects and people has long been considered an important building block for ubiquitous computing applications $[9,10]$. The most commonly available location technology today is the Global Positioning System (GPS). Unfortunately, GPS does not work well indoors, in urban canyons, or in similar areas with limited view of the sky. Instead, most research on indoor localization systems has been based on the use of short-range signals, such as 802.11 [3,7,13], Bluetooth [1], ultra sound [17], or infrared [18]. This paper shows that contrary to popular belief, an indoor localization system based on wide-area GSM fingerprints can achieve high accuracy, and is in fact comparable to an 802.11-based implementation.

This paper presents the first accurate indoor localization system based on fingerprinting of GSM signals. Fingerprinting relies on a training phase in which a radio map of the environment of interest is constructed by taking a series of radio measurements in multiple locations. A measurement records the strength at which signals emanating from a group of radio sources are heard at a given location. Once the training phase is complete, a client can estimate its location by matching the current measurement to the set of measurements collected in the training phase.

The key idea that makes accurate GSM-based indoor localization possible is the use of wide signal-strength fingerprints. The wide fingerprint includes the 6-strongest GSM cells and readings of up to 29 additional GSM channels, most of which are strong enough to be detected, but too weak to be used for efficient communication. The higher dimensionality introduced by the additional channels dramatically increases localization accuracy.

GSM-based indoor localization has several benefits: (i) GSM coverage far exceeds the coverage of 802.11 networks; (ii) the wide acceptance of cellular phones makes them ideal conduits for the delivery of ubiquitous computing applications. A localization system based on cellular signals, such as GSM, leverages the phone's existing hardware and removes the need for additional radio interfaces; (iii) because cellular towers are dispersed across the covered area, a cellular-based localization system would still work in situations where a building's electrical infrastructure has failed. Moreover, cellular systems are designed to tolerate power failures. For example, the cellular network kept working during the massive power outage that left most of the Northeastern United States and Canada in the dark in the Summer of 2003; (iv) GSM, unlike 802.11 networks, operates in a licensed band, and therefore does not suffer from interference from nearby devices transmitting on the same frequency (e.g., microwaves, cordless phones); and (v) the significant expense and complexity of cellular base stations ${ }^{1}$ result in a network that evolves slowly and is only reconfigured infrequently. While this lack of flexibility (and high configuration cost) is certainly a drawback for the cellular system operator, it results in a stable environment that allows the localization system to operate for a long period before having to be recalibrated.

We describe two case studies conducted over a course of two years. The first study examines the effects that fingerprint width and channel selection have on localization accuracy. We experimented with traces collected from three buildings located in Toronto

\footnotetext{
${ }^{1}$ A macro-cell costs $\$ 500,000$ to $\$ 1$ million. Micro-cells cost about a third as much, but a larger number is needed to cover the same area [16].
} 
and Seattle, which cover a wide spectrum of urban densities, ranging from a busy downtown core to a quiet residential neighborhood. Overall, the system achieves withinfloor median localization accuracy as low as $2 \mathrm{~m}$.

The second study examines the application of GSM localization technology to the specific problem of determining the floor in a tall building on which a user is located. Floorlevel localization is important in emergency situations where it can significantly reduce the area that rescue personnel have to canvas to locate individuals in large buildings. For example, the Empire State Building has a total floor area of 204,385 $\mathrm{m}^{2}$ spread over 102 floors. Floor-level localization reduces the area that needs to be searched by more than $99 \%$ to just $2000 \mathrm{~m}^{2}$ (about 18,000 $\mathrm{ft}^{2}$ ).

We collected traces in three tall buildings located in Toronto, Seattle and Washington DC using a commodity smart phone. Experimental results show that our system correctly identifies the floor up to $60 \%$ of the time and is within 2 floors up to $98 \%$ of the time. The system is robust, it works across a number of GSM network operators, when training and testing sets were collected by different smart phones of the same model and up to one month apart.

The rest of this paper is organized as follows. Section 2 describes related work. Section 3 gives a brief background on GSM and fingerprinting. Section 4 describes the localization algorithms we use. Sections 5 and 6 describe our two case studies and present their evaluation results. Finally, Section 7 concludes the paper.

\section{Related work}

This paper examines the effectiveness of GSM fingerprinting as an indoor localization technique. While this combination is new, indoor localization, radio fingerprinting and use of GSM for localization have all been explored before. We describe these efforts and key distinctions between these efforts and ours.

\subsection{Indoor localization}

While outdoor localization is almost exclusively performed using the Global Positioning System (GPS), indoor location systems have successfully employed a variety of technologies. The original Active Badge system [9] and follow on commercial systems like Versus [22] use infrared emitters and detectors to achieve 5-10 m accuracy. Both the Cricket [17] and the Bat [18] systems use ultrasonic ranging to estimate location. Depending on the density of infrastructure and degree of calibration, ultrasonic systems have accuracies between a few meters and a few centimeters. Most recently, ultra-wideband emitters and receivers have been used to achieve accurate indoor localization [21]. The common drawback of all of these systems is that they require custom infrastructure for every area in which localization is to be performed. As a result, these systems have not seen significant deployment outside of high-value applications like hospital process management. In contrast, GSM fingerprinting makes use of the existing GSM infrastructure, obviating the need for infrastructure investment and greatly increasing the possible area in which the system will work. This increases the likelihood of GSM fingerprinting achieving popular adoption. 


\subsection{Indoor localization using 802.11 fingerprinting}

Bahl and Padmanabhan [3] observed that the strength of the signal from an 802.11 access point does not vary significantly in a given location. They used this observation to build RADAR, a system that performed localization based on which access points would be heard where, and how strongly. This was the first fingerprinting system that showed that it is possible to localize a laptop in the hallways of a small office building within $2-3 \mathrm{~m}$ of its true location, using fingerprints from four 802.11 access points. There have been improvements to Radar's fingerprint matching algorithm that have improved accuracy $[2,13,19]$ and were able to differentiate between floors of a building with a high degree of precision [8]. In addition, commercial localization products have been built using 802.11 fingerprinting [20]. The differences between our work and 802.11 fingerprinting systems are primarily due to the differences between 802.11 and GSM that were outlined in Section 1: Due to higher coverage, GSM fingerprinting works in more places than 802.11 fingerprinting. Due to more stable infrastructure, 802.11 radio maps will degrade more quickly than GSM radio maps. Due to shorter range, 802.11 fingerprinting will be more accurate than GSM fingerprinting given the same number of radio sources.

\subsection{Localizing using GSM}

A number of systems have used GSM to estimate the location of mobile clients. The Place Lab system employed a map built using war-driving software and a simple radio model to estimate a cell phone's location with $100-150 \mathrm{~m}$ accuracy in a city environment [15]. The goal of Place Lab was to provide coarse-grained accuracy with minimal mapping effort. This is different, and complementary to our goal of doing accurate indoor localization given a detailed radio survey. Another distinction is that Place Lab used a cell phone platform that only programmatically exported the single associated cell tower.

Laitinen et al. [14] used GSM-based fingerprinting for outdoor localization. They have collected sparse fingerprints from the 6-strongest cells, achieving 67th percentile accuracy of $44 \mathrm{~m}$. Finally, Laasonen et al. used the transition between GSM cell towers to build a graph representing the places a user goes [12]. Like Place Lab, Laasonen's system used cell phones that only exported the single cell tower the phone was associated with. In contrast to the other systems we have mentioned, Laasonen's system did not attempt to estimate absolute location, but rather assigned locations symbolic names like Home and Grocery Store.

These previous efforts to use GSM for localization differ from the work reported in this paper in that they used narrow fingerprints that include the signal strength for the current cell $[12,15]$ or the 6 -strongest cells [14]. In contrast, we used wide fingerprints that include up to 29 different GSM channels in addition to the 6-strongest GSM cells, which significantly improve localization accuracy. In addition, previous efforts collected sparse fingerprints in outdoor environments, while we collected fingerprints indoors in a dense grid with $1.5 \mathrm{~m}$ granularity.

\section{Background}

This section first gives an overview of GSM and then describes radio fingerprinting. 


\subsection{GSM primer}

GSM is the most widespread cellular telephony standard in the world, with deployments in more than 210 countries by over 860 network operators [6]. In North America, GSM operates on the $850 \mathrm{MHz}$ and $1900 \mathrm{MHz}$ frequency bands. Each band is subdivided into $200 \mathrm{kHz}$ wide physical channels using Frequency Division Multiple Access (FDMA). Each physical channel is then subdivided into 8 logical channels based on Time Division Multiple Access (TDMA). There are 299 non-interfering physical channels available in the $1900 \mathrm{MHz}$ band, and 124 in the $850 \mathrm{MHz}$ band, totaling 423 physical channels in North America.

A GSM base station is typically equipped with a number of directional antennas that define sectors of coverage or cells. Each cell is allocated a number of physical channels based on the expected traffic load and the operator's requirements. Typically, the channels are allocated in a way that there is both increase in coverage and reduction in interference between cells. Thus, for example, two neighboring cells will never be assigned the same channel. Channels are, however, reused across cells that are far-enough away from each other so that inter-cell interference is minimized while channel reuse is maximized. The channel-to-cell allocation is a complex and costly process that requires careful planning and typically involves field measurements and extensive computer-based simulations of radio signal propagation. Therefore, once the mapping between cells and frequencies has been established, it rarely changes.

Every GSM cell has a special broadcast control channel (BCCH) used to transmit, among other things, the identities of neighboring cells to be monitored by mobile stations for handover purposes. While GSM employs transmission power control both at the base station and the mobile device, the data on the $\mathrm{BCCH}$ is transmitted at a full constant power. This allows mobile stations to compare signal strength of neighboring cells in a meaningful manner and choose the best one for further communication. It is these $\mathrm{BCCH}$ channels that we use for localization. In the rest of this paper, we refer to the BCCH channels simply as channels.

\subsection{Fingerprinting}

Two factors lead to the good performance of radio fingerprinting in the wireless band used by GSM and 802.11 networks. The first is that the signal strengths observed by mobile devices exhibit considerable spatial variability at the 1-10 $\mathrm{m}$ level. That is to say, a given radio source may be heard stronger or not at all a few meters away. The second factor is that these same signal strengths are consistent with time; the signal strength from a given source at a given location is likely to be similar tomorrow and next week. In combination, this means that there is a radio profile that is feature rich in space and reasonably consistent with time. Fingerprinting-based location techniques take advantage of this by capturing this radio profile for later reference.

Fingerprinting relies on a "training phase" in which a mobile device moves through the environment recording the strength of signals emanating from a group of radio sources (e.g., 802.11 access points, GSM base stations, FM radio [11] or TV stations). We refer to the physical position where the measurement is performed as a location, to the radio 
scan as a measurement and to the recording of the signal strength of a single source as a reading. That is, to build a radio map of the building, a mobile device takes a series of measurements in multiple locations of the building. Each measurement is composed of several readings; one for each radio source in range. The set of data recorded in a single location is also referred to as a training point. Since fingerprinting systems do not model radio propagation, a fairly dense collection of radio scans need to be collected to achieve good accuracy. The original RADAR experiments, for example, collected measurements every square meter on the average [3]. To achieve their advertised accuracy, the commercial 802.11 fingerprinting product from Ekahau [20] recommends a similar density.

Once the training phase is complete, a client can estimate its location by performing a radio scan (or equivalently collecting a testing point) and feeding it to a localization algorithm, which estimates the client's location based on the similarity of the signalstrength signatures between the testing and the training points. The similarity of signatures can be computed in a variety of ways, but it typically involves finding measurements in the training points that have the same radio sources with similar signal strengths.

\section{Localization algorithms}

In this section, we describe the localization algorithms we use in the rest of the paper. All our algorithms use the $K$-nearest neighbors [3] technique for matching fingerprints. Given a testing point and a list of training points, $K$-nearest neighbors estimates the location of the testing point in two stages.

First, the algorithm scans through all training points and calculates the Euclidean distance in signal space between the testing point and each of the training points. Then, the algorithm produces an estimate of the testing point's location by averaging the locations of the $K$ training points with the smallest Euclidean distance. To compute the Euclidean distance, the algorithm uses readings for all available radio sources in the fingerprint based on the assumption that the more radio sources are used the better the localization accuracy. For example, if a training fingerprint contains signal-strength readings for 3 sources $\left\{R_{1}^{t r}, R_{2}^{t r}, R_{3}^{t r}\right\}$ and a testing fingerprint has signal-strength readings for the same 3 sources $\left\{R_{1}^{\text {tst }}, R_{2}^{\text {tst }}, R_{3}^{\text {tst }}\right\}$ then the Euclidean distance between the two fingerprints will be calculated as:

$$
\sqrt{\left(R_{1}^{t r}-R_{1}^{\mathrm{tst}}\right)^{2}+\left(R_{2}^{t r}-R_{2}^{\mathrm{tst}}\right)^{2}+\left(R_{3}^{t r}-R_{3}^{\mathrm{tst}}\right)^{2}} .
$$

We implemented three localization algorithms which differ in the structure of their GSM fingerprints: onece $\iota$, uses the reading of the single-strongest GSM cell; cel $\iota$, uses readings of the 6-strongest GSM cells; and chann, uses readings from up to 35 GSM channels. For comparison purposes, we also implemented an algorithm dubbed 802.11, whose fingerprints include only readings from 802.11 access points.

Our algorithms use all available radio sources to compute the Euclidean distance between the testing and the training measurements. As it turns out, in practice, some of the radio sources may be either too noisy or too stable across different locations and including them in the calculation of the Euclidean distance may actually reduce localization accuracy. For example, if radio source 2 is identified for possible removal, its readings can 
be ignored, and the Euclidean distance between the training and the testing fingerprint can be calculated as:

$$
\sqrt{\left(R_{1}^{t r}-R_{1}^{\mathrm{tst}}\right)^{2}+\left(R_{3}^{t r}-R_{3}^{\mathrm{tst}}\right)^{2}} .
$$

The simplest approach for selecting the radio sources to be used for fingerprint matching would be to try all possible combinations of radio sources on the training data and pick the radio sources that result in the best performance. However, such search is exponential in the number of radio sources and therefore intractable. Instead, we used a greedy feature selection technique [4] to select a subset of highly relevant radio sources to be used in the Euclidean distance calculation. This greedy technique, albeit not optimal, has been shown to work well in practice [4]. The algorithm starts with a set that contains all available radio sources. At each step, the algorithm removes one radio source from the set. The radio source that is being removed is the radio source whose removal results in the largest increase in localization accuracy. The algorithm stops when removal of any radio source results in worse localization accuracy. In the rest of this paper, we refer to the version of chann that uses feature selection as chann ${ }_{f s}$.

\section{Within-floor localization study}

The within-floor localization study was conducted during the first half of 2005, and it examined the effect that fingerprint width and channel selection have on within-floor localization accuracy. In the rest of this section, we will describe our data collection process and data analysis, and then present our evaluation results.

\subsection{Data collection}

We collected measurements in two office buildings and one private detached house. The office buildings are the home to the Department of Computer Science of the University of Toronto and the Intel Research Seattle Lab. In the rest of this paper, we refer to these buildings as: University, Research Lab, and House.

University is a large $(88 \mathrm{~m} \times 113 \mathrm{~m})$ building with lecture rooms, offices and research labs, located in Toronto's busy downtown core. Since we had no access to the offices, we collected training points in the hallways of the 7th floor of the building. Research Lab is a medium size $(30 \mathrm{~m} \times 30 \mathrm{~m})$ building, located in Seattle's commercial midtown. Space inside the building is partitioned with semi-permanent cubicles. Due to access restrictions, we collected measurements only from the 5th floor of the building. House is a wooden structure $(18 \mathrm{~m} \times 6 \mathrm{~m})$, located in a quiet residential neighborhood of Seattle. We collected measurements on the first floor of the house.

We collected both 802.11 and GSM fingerprints using a laptop running Windows XP. To collect 802.11 fingerprints, we used an Orinoco Gold wireless card configured in active scanning mode, where the laptop periodically transmits probe requests and listens to probe responses from nearby 802.11 APs.

We collected GSM fingerprints using a Sony Ericsson GM28 GSM modem, shown in Fig. 1, which operates as an ordinary GSM cell phone, but exports a richer 


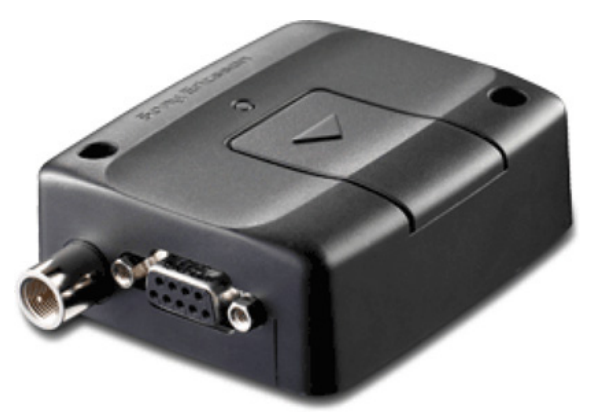

Fig. 1. Sony Ericsson GM28 modem.

Table 1

Average signal strength ( $\mathrm{db} \mathrm{m})$ for cells and channels

\begin{tabular}{llll}
\hline & University (downtown) & Research lab (midtown) & House (residential) \\
\hline Cells & -87.69 & -76.74 & -88.35 \\
Channels & -96.41 & -102.19 & -105.27 \\
\hline
\end{tabular}

programming interface. The GSM modem provides two interfaces for accessing signalstrength information: cellsAPI and channelsAPI. ${ }^{2}$ The cellsAPI interface reports the cell ID, signal strength, and associated channel for the $n$-strongest cells. While the modem's specification does not set a hard bound on the value of $n$, in practice in the 3 environments we measured $n$ was equal to 6 . The channelsAPI interface simultaneously provides the signal strength for up to 35 channels, 13 of which can be specified by the programmer, with up to 22 additional channels picked by the modem itself. In practice, 6 of the 35 channels typically correspond to the 6-strongest cells. Unfortunately, channelsAPI reports signal strength but does not report cell IDs. We speculate that the cell ID information for other than the 6-strongest cells cannot be determined because the IDs of the cells may not be extractable from the weak signals with high enough reliability.

Table 1 shows the average signal strength returned by the cellsAPI and channelsAPI interfaces. As expected, the average signal strength reported by cellsAPI is significantly higher than the average signal strength reported by channelsAPI. Note that the average signal strength reported by the channelsAPI interface is close to the modem's stated receiver sensitivity ${ }^{3}$ of $-102 \mathrm{db}$ m. Efficient GSM communication requires an SNR higher than $-90 \mathrm{db}$.

The lack of cell ID information for some channels raises the possibility of aliasing, i.e., a situation when two or more cells transmitting simultaneously on the same channel appear to be a single radio source and therefore cannot be differentiated. In the extreme case, a fingerprinting system that relies exclusively on channel-based data may suffer from worldwide aliasing. Because channels are reused throughout the world, measurements

\footnotetext{
2 The terms cellsAPI and channelsAPI are used to simplify presentation. In practice, the cellsAPI correspond to $\mathrm{AT} * \mathrm{E} 2 \mathrm{EMM}=1$ command and the channelsAPI correspond to the AT*E2NBTS? command on the GM28 GSM modem, respectively.

3 In practice, the modem reports signal strength as low as $-115 \mathrm{db}$.
} 


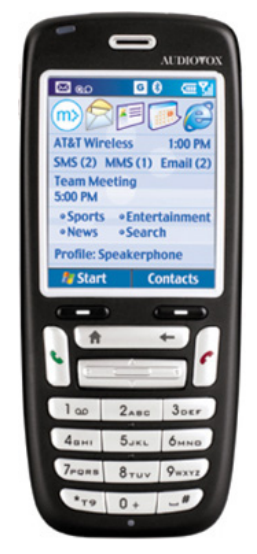

Fig. 2. Audiovox SMT 5600 phone.

taken in two far-away locations may produce similar fingerprints. To alleviate the aliasing problem, we combine the information returned by the cellsAPI and channelsAPI interfaces into a single fingerprint. We then restrict the set of fingerprints to which we compare a testing point to fingerprints that have at least one cell ID in common with the testing point. This practice effectively differentiates between fingerprints from our three indoor environments.

As we show in Section 5.3, our localization system based on wide GSM fingerprinting significantly outperforms GSM fingerprinting based on the 6-strongest cells, and is comparable to 802.11-based fingerprinting. This is because our fingerprints are wide (have many readings), and therefore, in order for the aliasing to reduce accuracy, many readings in the fingerprints of distant locations need to match, which is highly unlikely in practice.

We developed a simple Java-based application to assist us in the process of gathering fingerprints. To record a fingerprint, we first identify the current position by clicking on a map of the building. The application then records the signal strengths reported by the 802.11 card and the cellsAPI and channelsAPI interfaces of the GSM modem. To collect the measurements, we placed the laptop on an office chair and moved the chair around the building. While primitive, this setup assures measurements collected at a constant height. In all three indoor environments, we collected 802.11 and GSM fingerprints for points located $1-1.5 \mathrm{~m}$ apart. We collected 2 measurements per location, waiting $5 \mathrm{~s}$ between the scans (the default value according to the modem specification). Overall, we collected measurements at 154 locations in the University, 181 locations in the Research Lab and 44 locations in the House.

\subsection{Data analysis}

In this section, we first investigate the stability of GSM and 802.11 signals over time at a single location and then show the distribution of the fingerprint widths as recorded in the University building.

To compare the stability of GSM and 802.11 signals, we recorded the signal strength of nearby 802.11 access points (AP) and 6-strongest GSM cells at several locations in one of the buildings that houses the Department of Computer Science at the University of 


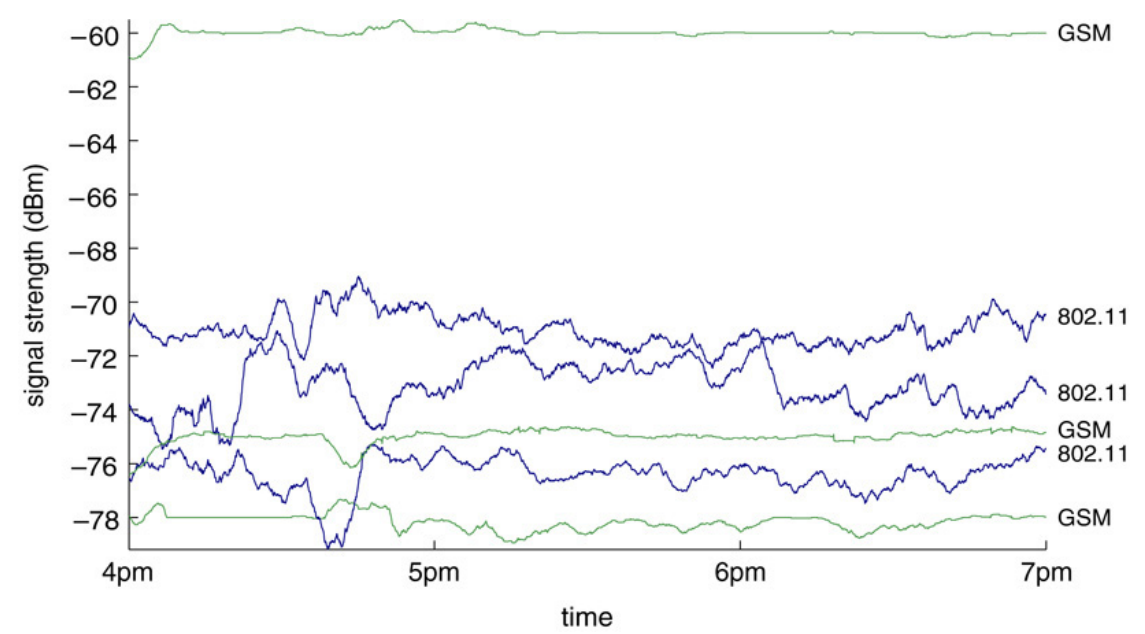

Fig. 3. 802.11 and GSM signal stability over time.

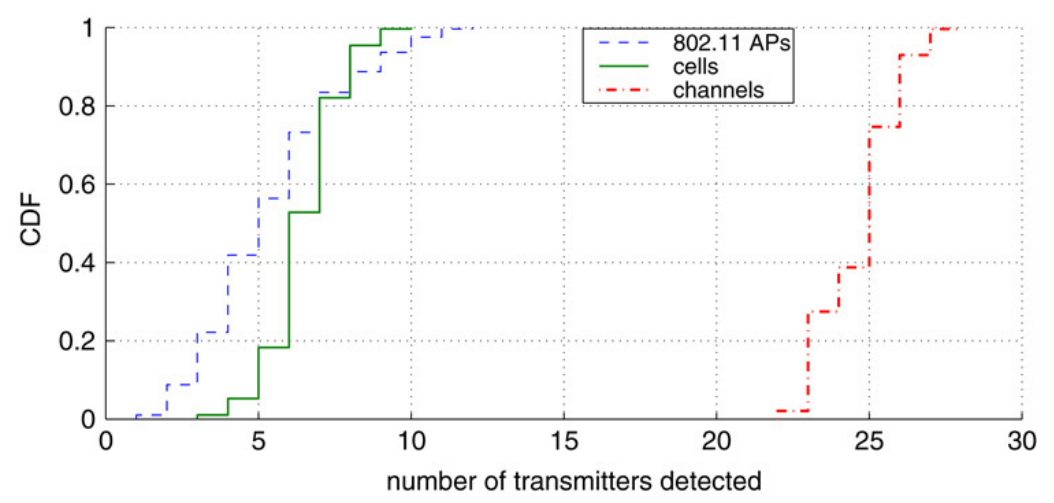

Fig. 4. Cumulative distribution of the fingerprint width at the University building. Shown are fingerprints based on 802.11 AP, GSM cells, and GSM channels.

Toronto. Fig. 3 shows a $3 \mathrm{~h}$ segment of the signal-strength measurements at a location on the fifth floor of the building during a workday afternoon. The plot shows the 3-strongest GSM cells and the 3-strongest 802.11 APs. GSM signals appear to be more stable than 802.11 signals. We believe that this is because 802.11 uses crowded unlicensed $2.4 \mathrm{GHz}$ band, and therefore suffers from interference from nearby appliances such as microwaves and cordless phones. An analysis of GSM signal stability over longer periods of time and under different weather conditions (e.g., rain, snow, fog) is left for future work.

Fig. 4 plots the cumulative distribution function (CDF) of the fingerprint width at the University building. Fingerprints based on 802.11 AP, GSM cells, and GSM channels are shown in the figure. The figures for the Research Lab and the House show similar patterns and are therefore not included. The median widths of 802.11 AP and GSM cells fingerprints are 5 and 6, respectively. In contrast, the median width of GSM channel fingerprints is 25 . We will show in the next section that the larger fingerprint has a dramatic effect on localization performance. 
Table 2

Within-floor 50\% and 95\% localization error (m)

\begin{tabular}{|c|c|c|c|c|c|c|}
\hline & \multicolumn{2}{|c|}{ University (downtown) } & \multicolumn{2}{|c|}{ Research lab (midtown) } & \multicolumn{2}{|c|}{ House (residential) } \\
\hline & 50-percentile & 95-percentile & 50-percentile & 95-percentile & 50-percentile & 95-percentile \\
\hline 802.11 & 4.78 & 19.92 & 2.20 & 11.40 & 3.43 & 9.66 \\
\hline $\operatorname{chann}_{f s}$ & 3.02 & 17.92 & 2.50 & 10.70 & 1.94 & 7.79 \\
\hline chann & 4.07 & 27.79 & 3.40 & 16.35 & 3.36 & 12.41 \\
\hline cell & 8.02 & 32.14 & 4.82 & 15.78 & 3.41 & 10.79 \\
\hline onecell & 14.64 & 55.51 & 8.39 & 20.45 & 4.85 & 13.36 \\
\hline random & 30.43 & 65.72 & 10.40 & 20.06 & 6.21 & 14.35 \\
\hline
\end{tabular}

\subsection{Evaluation results}

The results reported in this section were obtained using leave-one-out cross-validation method, which takes one point at a time out of the training set and uses it as the testing point. This technique is similar to that used by Bahl [3].

Table 2 summarizes the localization errors for the 5 algorithms introduced in Section 4 for the three indoor environments. For each building, the table shows the 50-percentile and the 95-percentile localization errors, calculated as the Euclidean distance between the actual and predicted location of the point within a floor. Table 2 also presents results for random, an algorithm that arbitrarily picks a point from the training set data and assigns its location as the predicted location. random provides a lower bound on the performance of localization systems for a given floor and building. The localization error in random depends on the size of the floor, which accounts for the difference in its localization error across buildings.

Across the three buildings, 802.11 achieves median accuracy between 2.2 and $4.8 \mathrm{~m}$. These results are consistent with results previously reported in the literature. Differences in accuracy between buildings reflect discrepancies in the granularity of the measurement grid which varied between 1 and $1.5 \mathrm{~m}$, the difference in floor areas, and the difference in the number of points taken on each floor.

There are large differences in the performance of the various GSM-based algorithms. chann and chann $f_{s}$ outperform cell and onecell in all cases. Across the three buildings, chann $_{f s}$ achieves median accuracy between 1.94 and $3.02 \mathrm{~m}$, which outperforms 802.11 in the University building and the House.

The strong performance of chann demonstrates the advantage of wide fingerprints, i.e., including measurements from a large number of channels rather than just the 6-strongest cells. Moreover, the significant accuracy improvement of chann $f s$ over chann shows that selecting a subset of highly relevant channels for fingerprint matching has an important effect on systems performance.

Fig. 5 shows the cumulative distribution (CDF) of the localization error of all algorithms for the University building. Most remarkable is the closeness with which $\operatorname{chann}_{f s}$ approximates 802.11, and the large difference in performance between chann $_{f s}$ and cell. 


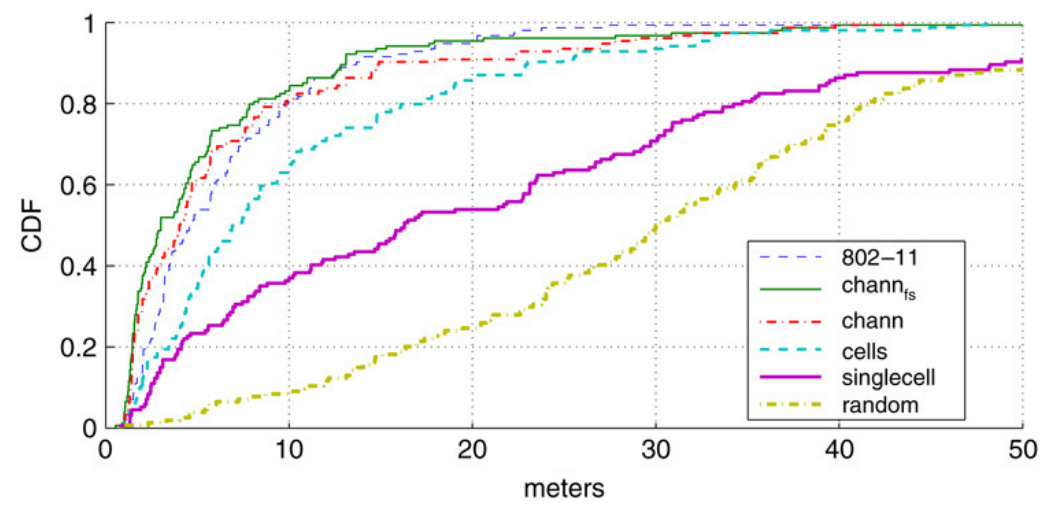

Fig. 5. CDF of the localization error in the University building.

\subsection{Sensitivity analysis}

In this section, we analyze the best GSM performer, chann $f_{s}$, in more detail. Specifically, we test the localization accuracy of $\operatorname{chann}_{f s}$ as a function of the number of channels used and the number of measurements collected per location.

\subsubsection{Number of channels}

Fig. 6 plots the median localization error as a function of the number of channels used. Increasing the number of channels results in a larger fingerprint, which allows for a better comparison between neighboring points and therefore for improved localization accuracy. The channels picked are sorted by popularity (i.e., the number of fingerprints on which a specific channel appears). For example, the median localization error for 6 channels, corresponds to an algorithm where the 6 (fixed) most popular channels are picked from the training set. Notice that the accuracy of the algorithm that picks the 6 most popular channels is lower than that of the cell algorithm. This is because the cell algorithm picks the 6-strongest cells for each measurement, which may result in much larger fingerprint vector (e.g., completely different 6 cells may be picked in two distant locations, increasing the fingerprint vector to 12 entries).

\subsubsection{Number of measurements per location}

Although all the results reported so far were based on the average of 2 measurements per location, we actually obtained 10 measurements per location for the University building dataset. However, experiments varying the number of measurements per location between 2 and 10 showed virtually no difference in the accuracy of the algorithms. This is because our readings are stable and therefore adding more measurements per location does not improve localization accuracy.

\section{Floor-level localization study}

The floor-level localization study was conducted during the first half of 2006. The study examined the application of GSM localization technology to the specific problem of 


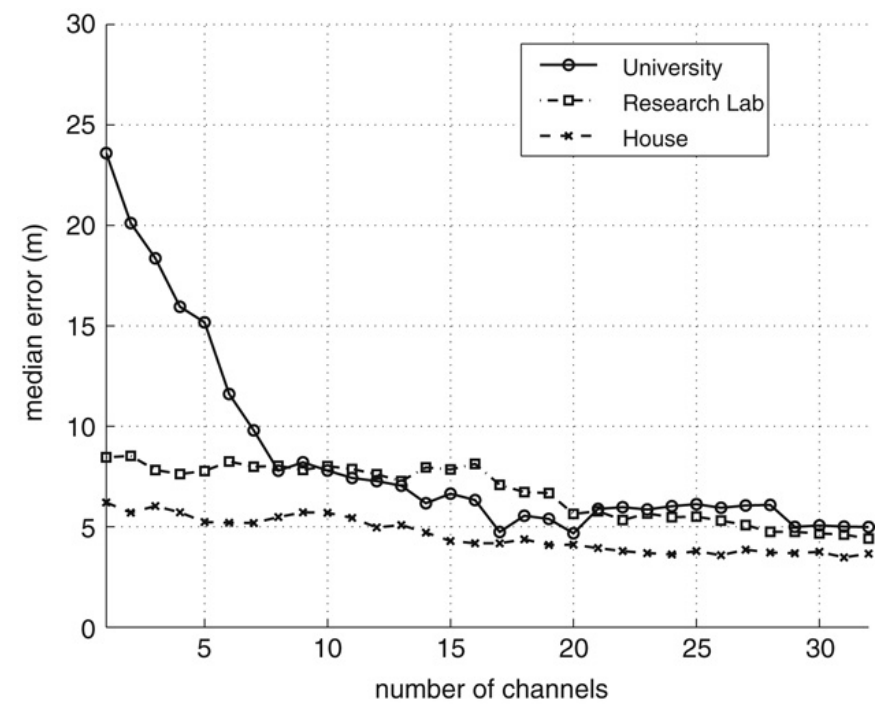

Fig. 6. Localization error as a function of fingerprint size.

determining the floor on which a user is located in a tall building. As part of the study, we implemented a system called SkyLoc that runs on commodity off-the-shelf phones. In the rest of this section, we describe the SkyLoc system, give details about our data collection process and then present our evaluation results.

\subsection{SkyLoc}

SkyLoc is a system that runs on a GSM mobile phone and determines the floor within a building on which a user is located. The system is implemented in C\# and was tested on an AudioVox SMT 5600 phone shown in Fig. 2. The phone runs the Windows Mobile 2003 operating system. SkyLoc measures GSM environment by continuously taking GSM measurements at a rate of 1 measurement per second. Each measurement contains signalstrength information for 7 GSM cells and up to additional 15 GSM channels. The SkyLoc system has two components: a data collection application called PlaceLogger and a fingerprint matching and visualization application called PlaceLocator.

PlaceLogger supports creating a hierarchical representation of places visited by a user and then collecting GSM measurements for these places (e.g., floors in a building). Fig. 7 shows a screen shot of the PlaceLogger interface. The top of the screen shows a tree of the places entered by a user. In our case, the tree has a depth of 2 , having the names of buildings as root nodes and the floors as leaf nodes. PlaceLogger supports scrolling through the nodes, adding new nodes, deleting nodes or selecting nodes. Once the user selects a node, she can press the Enter Place button to start the data collection process. To stop the data collection, the user presses the Exit Place button. The lower part of the screen shows the name of the place for which measurements are being collected and the number of measurements collected so far at this place.

PlaceLocator shows the same hierarchical view of places recorded by PlaceLogger. However, once loaded, PlaceLocator continuously takes GSM measurements, matches 


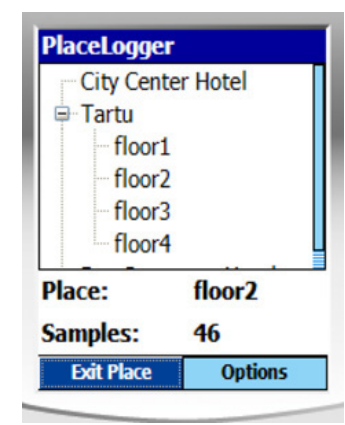

Fig. 7. PlaceLogger.

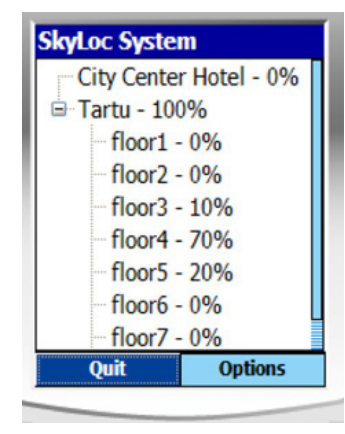

Fig. 8. PlaceLocator.

them to the training measurements collected by PlaceLogger, and presents its floor number prediction to the user. The results are represented in a hierarchical manner. First, the probability of being at a leaf node is calculated and then these probabilities are propagated up the tree to the roots. The screen shot of PlaceLocator is shown in Fig. 8. The Options menu allows selecting various parameters for the matching algorithm.

Currently, SkyLoc is implemented as a stand alone application running on the mobile phone. The phone calculates the current location locally and transmits it to the emergency services as required. The advantage of this approach is that it provides a fast way to get the system up and running today. However, we envision our system being eventually adapted, deployed and maintained by network operators or other third parties. In this scenario, when a user dials for emergency response, the phone takes a few measurements and transmits them to a server, which will calculate the phone's current location and forward this location to the emergency services.

Our initial experience with SkyLoc is very encouraging. Collecting training data for a new building is quite easy and not very time consuming. Moreover, as we show in Section 6.3, the system has good accuracy.

\subsection{Data collection}

We collected fingerprints in the hallways of 3 buildings: (a) City Center Hotel; (b) University Hotel; and (c) Tartu building. The buildings are shown in Fig. 9. City Center Hotel is a 9-storey building, located in a quiet midtown residential area of Washington DC. 


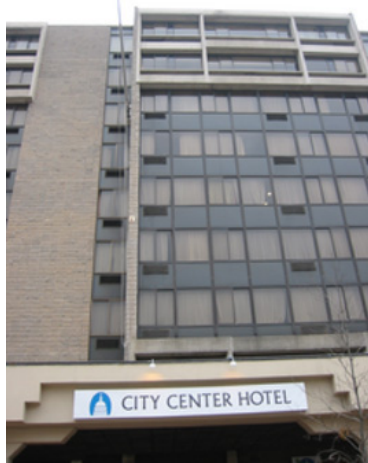

(a) City Center Hotel, Washington DC.

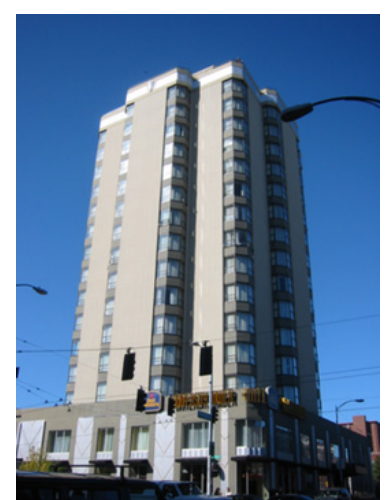

(b) University Hotel, Seattle, Washington.

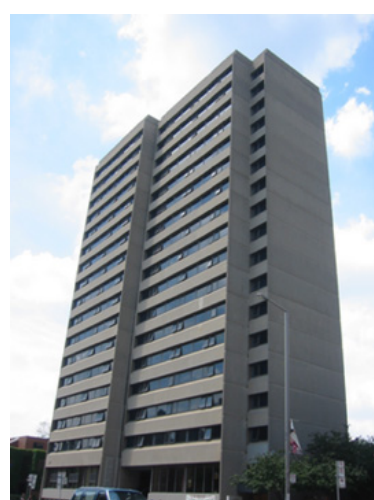

(c) Tartu Building, Toronto, Ontario.

Fig. 9. The tall multi-floor buildings where the data was collected.

Table 3

Characteristics of the 3 buildings under study

\begin{tabular}{lclr}
\hline & City Center Hotel & University Hotel & Tartu \\
\hline Number of floors & 9 & 12 & 16 \\
Fingerprints per floor & 110 & 30 & 130 \\
Training file size (kB) & 66 & 33 & 320 \\
\hline
\end{tabular}

University Hotel is a 12-storey building located in a midtown commercial area of Seattle. Finally, Tartu is a 16-storey building, located in downtown Toronto. Taking fingerprints in different cities and different urban environments allowed us to assess the robustness of SkyLoc across environments.

Table 3 summarizes the number of fingerprints collected per floor for each of the buildings. ${ }^{4}$ The different number of fingerprints collected per floor is the result of us increasing the number of training and testing fingerprints collected with every new building in the hope of achieving even better localization results. Ironically, as we show in Section 6.3.2, the number of training fingerprints has little bearing on the localization accuracy.

We collected fingerprints for several available network operators simultaneously (using different phones), scanning the network every second. Once we started the data collection, we walked with an average speed of about $2 \mathrm{~m} / \mathrm{s}$ on each of the floors, collecting fingerprints. We collected data during the day hours when people were present on the floors. Whereas this practice may have a negative affect over SkyLoc's measured performance, we believe that it provides a more realistic estimate of the system's expected performance under real world conditions.

To investigate the effects of using different phones for training and testing and the effects of separating the training and testing in time, we collected additional fingerprints in City

\footnotetext{
${ }^{4}$ The buildings are sorted by height.
} 


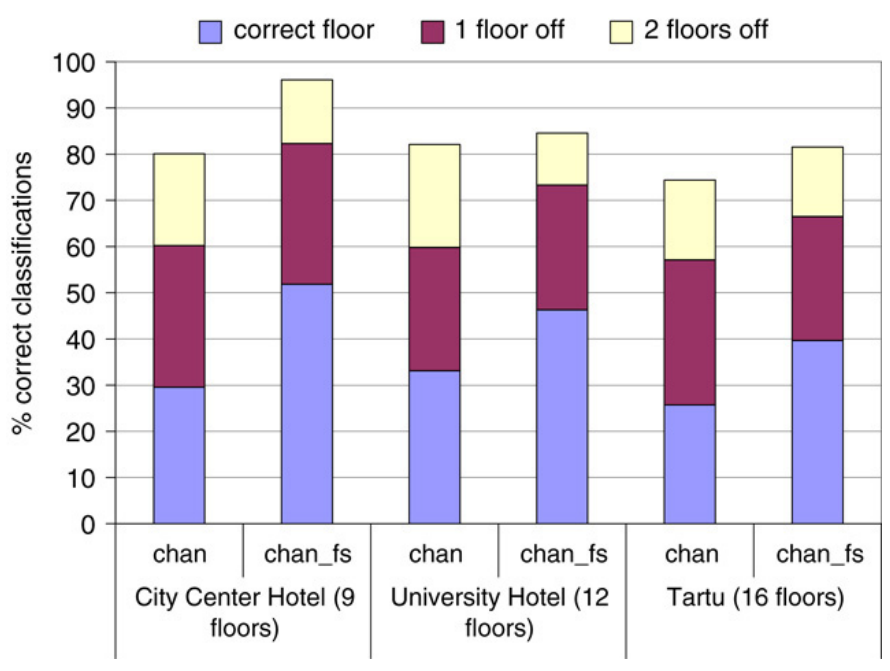

Fig. 10. Accuracy results across all buildings.

Center Hotel two days after the initial fingerprints were collected and in University Hotel a month after the initial fingerprints were collected. In both cases, we collected fingerprints using different instances of the AudioVox phone.

\subsection{Evaluation results}

In this section, we evaluate how accurately the chann and chann $f_{s}$ algorithms presented in Section 4 can differentiate between floors in tall multi-floor buildings. As described in Section 6.2, we collected separate traces for training and testing data in each of the 3 buildings and we used those traces as input to the algorithms. Unless otherwise specified, the delay between collecting training and testing data on each floor was between one to two hours.

Fig. 10 summarizes the accuracy with which the algorithms can correctly determine the current floor, be it 1 floor off (predict the adjacent floor as the correct floor) and be it 2 floors off. The chann $f_{s}$ algorithm performs better than chann, achieving $51 \%$ correct floor classifications and $96 \%$ of correct classifications within 2 floors for the City Center Hotel. The chann algorithm trails behind with $30 \%$ correct floor classifications and $80 \%$ of correct classifications within 2 floors for the same building. We found that the main reason for the low performance of the chann algorithm is that, in some cases, the training and testing fingerprints collected on the same floors contained readings from partially different sets of base stations. Although the presence of people on the floors may have increased the discrepancy, we believe that the main reason for the discrepancy lies in the way a mobile phone picks cells and channels to listen to. According to the GSM specification [6], the phone gets the list of neighboring cells to listen to from the associated cellular tower, which is not necessarily, but often, the tower with the strongest signal strength. The way the phone picks the associated tower depends on the strength and quality of the signal received from neighboring cells and on additional parameters, such as the time the phone was associated with the cell. Overall, this occasionally results in the phone picking different 
associated cells for the training and testing data on the same floor, which in turn results in lower localization accuracy. Fortunately, even when the associated cells are different, some of the neighboring cells and channels are still the same. It is these common cells that the feature selection algorithm uses to achieve higher localization results.

One might expect to observe better localization accuracy for lower buildings because the fewer floors a building has, the lower the probability of getting the current floor wrong. For example, in a building with only 3 floors even an algorithm that guesses the current floor at random will be correct roughly $33 \%$ of the time. The results support this hypothesis, but to a small extent. For example, chann ${ }_{f s}$ achieved $96 \%$ accuracy within 2 floors in City Center Hotel, $84 \%$ in University Hotel and $82 \%$ in Tartu. Data analysis showed that this is mainly due to the fact that when the classifier is wrong, it is usually wrong within 1 or 2 floors and therefore increasing the number of floors may not necessarily affect accuracy. For instance, the radio environment on a 2 nd floor might be similar to the one on the 3rd or the 4th floor, but it is as drastically different from the one on the 10th floor as it is from the one on the 20th.

\subsubsection{Windowing}

The previous section showed localization results for testing fingerprints classified independently of one another. In practice, the classification decision need not necessarily be made on a single testing fingerprint, but may be made based on a stream of testing fingerprints.

We implemented a simple algorithm that makes the classification decision based on a fixed-size sliding window of testing measurements. For example, if the window size is 10 , the classification decision is based on the current measurement and the nine preceding measurements. The windowing algorithm first classifies each measurement in the window individually, and then selects the current floor as the most frequently appearing floor among the individual classifications.

Fig. 11 shows the classification accuracy for the chann ${ }_{f s}$ algorithm when the number of testing fingerprints in the window varies from 1 to 20 . The classification accuracy increases with the window size for all buildings, reaching $98 \%, 90 \%$ and $82 \%$ of correct classifications within 2 floors for the City Center Hotel, University Hotel and Tartu building, respectively. Although in areas with large number of misclassifications, windowing does not help much, it does help to remove outliers when the overall performance is good, and we believe that it should be used by localization systems.

\subsubsection{Sensitivity analysis}

In this section, we quantify the sensitivity of the classification accuracy to different network operators, the collection of training and testing data with different phones of the same model, separating the training and testing in time, and the number of training fingerprints collected per floor.

Fig. 12 shows the localization results for the University Hotel for different network operators. The results suggest that our system works across different network providers, as there seems to be no significant difference in terms of achievable accuracy between different network operators. The results for City Center Hotel and Tartu buildings (not included) show a similar trend. 


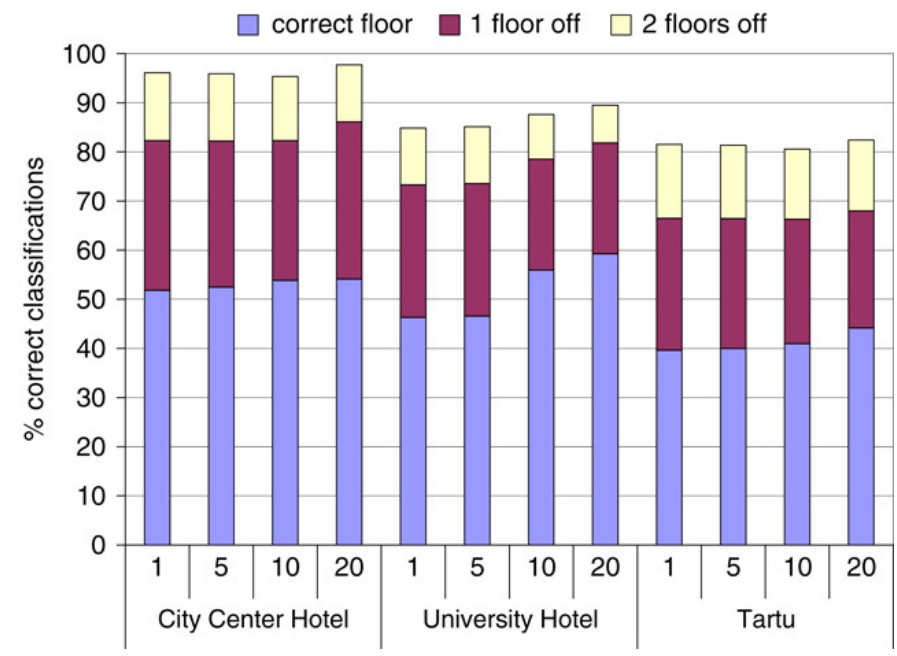

Fig. 11. The effect of windowing on top of the chann $f_{s}$ algorithm.

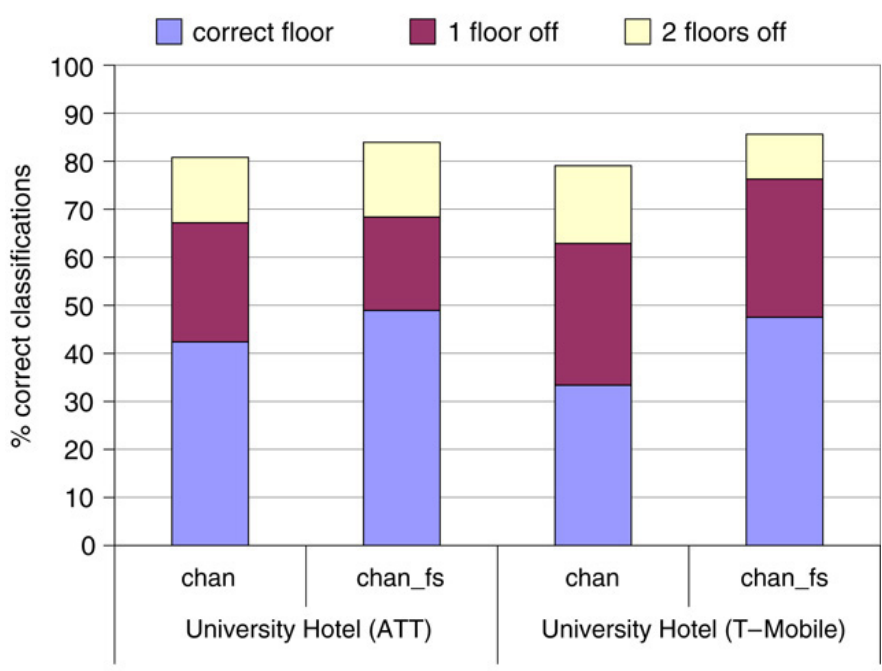

Fig. 12. The effect of varying network operators.

Fig. 13 shows the effect of taking the training and testing fingerprints with different phones of the same model in the University Hotel and Tartu building. The results confirm that taking fingerprints with a different phone does not significantly affect localization accuracy. In the University Hotel, the percentage of correct floor classifications has reduced from $46 \%$ to $42 \%$ and for the Tartu building it has reduced from $39 \%$ to $30 \%$. Interestingly, although the percentage of correct floor classifications within 2 floors has slipped $8 \%$ in the University Hotel, the percentage actually rose $2 \%$ in the Tartu building.

Fig. 14 shows the effect of taking the training and testing fingerprints 2 days and a month apart for the City Center Hotel and the University Hotel. The results show that taking testing fingerprints a few days or even a month apart does not significantly 


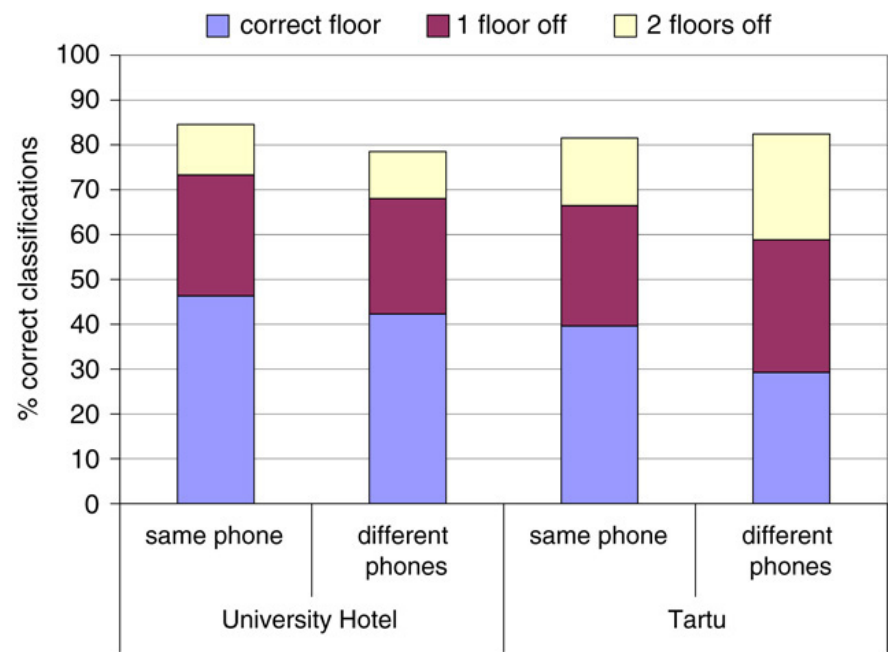

Fig. 13. The effect on chann $f_{s}$ of collecting testing and training measurements with different phones.

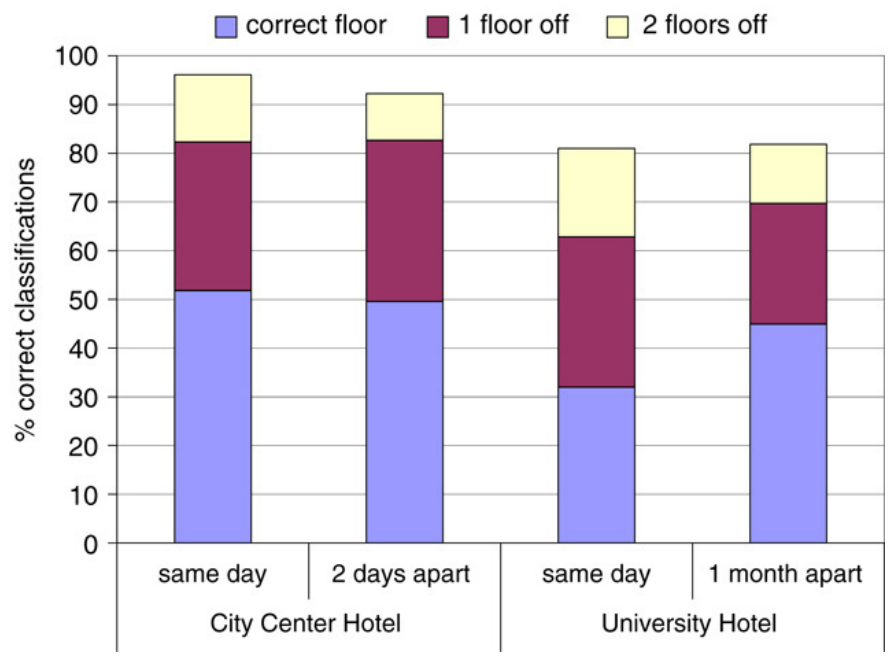

Fig. 14. The effect on chann $f_{s}$ of taking the training and testing fingerprints 2 days and a month apart.

affect localization accuracy. For the City Center Hotel, the percentage of correct floor classifications within 2 floors slipped from $96 \%$ to $93 \%$, and the number of correct floor classifications has reduced from $52 \%$ to $50 \%$. For the Tartu building, the performance was similar, with correct floor classifications slipping from $46 \%$ to $45 \%$.

Fig. 15 shows the effect of reducing the number of training fingerprints collected per floor for each of the 3 buildings. The figure plots the percentage of correct floor classifications as a function of the percentage of training fingerprints used. For example, $50 \%$ of the testing points were classified correctly in the City Center Hotel with both onefourth and one-tenth of the originally collected training points. Surprisingly, the reduction in accuracy among $100 \%$ of training fingerprints to only $10 \%$ is small across all buildings. 


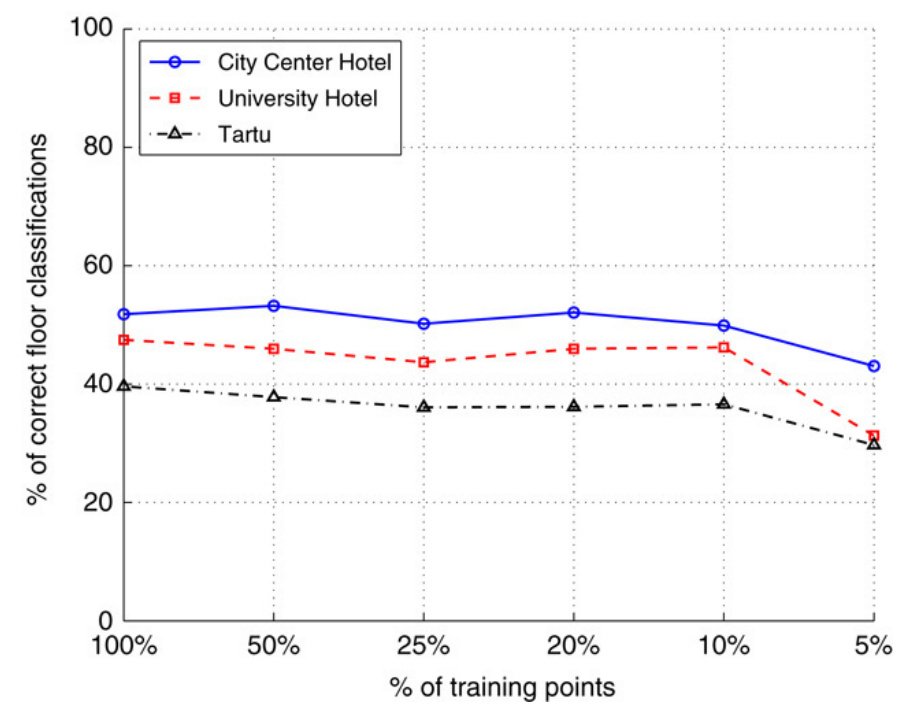

Fig. 15. The effect of reducing the number of training fingerprints.

This is a very encouraging result because it means that only a small number of training fingerprints need to be collected per floor, or in other words we could train any of the buildings under study in less than $30 \mathrm{~min}$ and still achieve good localization results.

\subsubsection{Performance evaluation}

In this section, we present our performance evaluation of the SkyLoc system in terms of memory and storage footprint and localization run times.

The amount of training data that needs to be stored on the phone depends on the building size. The taller the building and the larger the floor size, the larger the training file. Our current prototype stores the data in a raw text format without performing any storage optimizations. The training file sizes are summarized in Table 3. It follows that with the current flash card sizes of $1 \mathrm{~GB}$ it is possible to store training files of more than 7000 buildings on a single card. Moreover, the training files may be stored in an archive file (e.g., zip) most of the time and extracted only on demand. This optimization reduces the storage requirement on the phone by an order of magnitude (archiving the $320 \mathrm{kB}$ training file from the Tartu building produces a $30 \mathrm{kB}$ zip file). Note that instead of storing all fingerprint maps on the phone, the phone may be able to simply download them upon entering a building. The building may be identified by either using a GPS receiver if one is available on the phone or through a GSM-based wide-area localization system [5].

The SkyLoc application takes about $200 \mathrm{kB}$ storage space including all the necessary libraries. When loaded it takes about $1600 \mathrm{kB}$ of memory, plus any additional memory needed for the training data. For example, the SkyLoc application and the Tartu building training file take about $2 \mathrm{MB}$ of memory out of the $32 \mathrm{MB}$ available on our AudioVox SMT 5600 phone.

Next, we measured the scalability of SkyLoc in terms of the time it takes to locate a single testing fingerprint on AudioVox's $200 \mathrm{MHz}$ Texas Instruments OMAP processor. Determining the location of a fingerprint requires matching the fingerprint against the 
current training set. Note that in order to locate a fingerprint there is no need to match the fingerprint to all fingerprints stored on a phone, but only to a set of relevant fingerprints. One approach that we found to work well in practice is matching only against training fingerprints that have at least one cell ID in common with the current testing fingerprint.

We conducted a series of experiments, each time varying the training file size and measuring the time it takes to locate a single testing fingerprint. On an average, it takes $0.002 \mathrm{~s}$ to match a single testing fingerprint to a single training fingerprint or equivalently the phone can match a testing fingerprint to 500 training fingerprints in a second. For instance, in the University Hotel, it takes about $0.72 \mathrm{~s}$ to localize a fingerprint. We are planning to develop faster fingerprint matching techniques in the future.

\subsection{Discussion and recommendations}

Should floor identification be added to the E911/E112 specifications, we recommend that regulatory bodies start with the requirement of "within 2 floors of the actual floor number $95 \%$ of the time". We have demonstrated that the 2 floor- $95 \%$ goal is achievable in software on mobile phones and thus it represents a good starting point for any discussions of extending regulations of the third dimension. While a lower error margin might be necessary for some E911/E112 scenarios, we believe that regulation works best if it starts with what is possible and then evaluates if it is sufficient.

The largest barrier to wide-scale adoption of our approach is probably the requirement to gather training data for each building. However, we believe that that such a calibration could be made a part of the regulated zoning procedures for large buildings and is probably low overhead compared to the many stringent building codes and maintenance procedures already in place for a multi-floor building like elevator maintenance and emergency exit lighting and signage. The fact that calibration maps seem capable of being transferred between devices without significantly impacting accuracy also supports this deployment model.

\section{Conclusions}

This paper demonstrated that accurate indoor GSM-based localization is possible thanks to the use of wide signal-strength fingerprints that include readings of up to 29 GSM channels in addition to the 6-strongest cells. We also showed that the localization performance can be further improved by carefully selecting a subset of highly relevant channels to be used for fingerprinting matching.

We presented our experience and evaluation results from two studies, conducted in the first halves of 2005 and 2006. The first study examined how fingerprint width and channel selection affect within-floor localization accuracy. We evaluated our system based on traces collected in three buildings located in the Toronto and Seattle metropolitan areas. Our GSM-based indoor localization system achieves a median accuracy ranging from 1.94 to $4.07 \mathrm{~m}$.

The second study examined the application of GSM localization technology to the specific problem of determining the floor in a tall building on which a user is located. We presented evaluation results from three multi-floor buildings located in Washington 
DC, Seattle, and Toronto. Our system identified the floor correctly in up to $60 \%$ of the cases and is within 2 floors in $98 \%$ of the cases. Our system is robust; it works for different network operators, when the training and testing sets were collected with different phones of the same model and up to one month apart.

\section{References}

[1] L. Aalto, N. Gothlin, J. Korhonen, T. Ojala, Bluetooth and WAP push based location-aware mobile advertising system, in: Proceedings of the 2nd International Conference on Mobile Systems, Applications, and Services, ACM Press, 2004.

[2] P. Bahl, A. Balachandran, V. Padmanabhan, Enhancements to the RADAR user location and tracking system, Microsoft Research, Technical Report, Feb. 2000.

[3] P. Bahl, V.N. Padmanabhan, RADAR: An in-building RF-based user location and tracking system, in: Proceedings of INFOCOM, 2000.

[4] A. Blum, P. Langley, Selection of relevant features and examples in machine learning, Journal on Artificial Intelligence (1997)

[5] M.Y. Chen, T. Sohn, D. Chmelev, D.H.J. Hightower, J. Hughes, A. LaMarca, F. Potter, I. Smith, A. Varshavsky, Practical metropolitan-scale positioning for GSM phones, in: Proceedings of the Eighth International Conference on Ubiquitous Computing, Irvine, CA, 2006.

[6] J. Eberspacher, H.-J. Vogel, C. Bettstetter, GSM Switching, Services and Protocols, John Wiley \& Sons Ltd, 2001.

[7] E. Elnahrawy, X. Li, R. Martin, The limits of localization using signal strength: A comparative study, in: Proceedings of the 1st IEEE International Conference on Sensor and Ad Hoc Communications and Networks, Santa Clara, CA, 2004.

[8] A. Haeberlen, E. Flannery, A.M. Ladd, A. Rudys, D.S. Wallach, L.E. Kavraki, Practical robust localization over large-scale 802.11 wireless networks, in: Proceedings of the Tenth ACM International Conference on Mobile Computing and Networking, Philadelphia, PA, 2004.

[9] A. Hopper, A. Harter, T. Blackie, The active badge system, in: Proceedings of INTERCHI-93, Amsterdam, The Netherlands, 1993.

[10] T. Kindberg, A. Fox, System software for ubiquitous computing, IEEE Pervasive Computing 1 (1) (2002) $26-35$.

[11] J. Krumm, G. Cermak, E. Horvitz, RightSPOT: A novel sense of location for smart personal objects, in: Proceedings of the Fifth International Conference on Ubiquitous Computing, 2003.

[12] K. Laasonen, M. Raento, H. Toivonen, Adaptive on-device location recognition, in: Proceedings of the Second International Conference on Pervasive Computing, Springer-Verlag, 2004.

[13] A. Ladd, K. Bekris, G. Marceau, A. Rudys, L. Kavraki, D. Wallach, Robotics-based location sensing using wireless ethernet, in: Proceedings of the Tenth ACM International Conference on Mobile Computing and Networking, MOBICOM, 2002.

[14] H. Laitinen, J. Lahteenmaki, T. Nordstrom, Database correlation method for GSM location, in: Proceedings of the 53rd IEEE Vehicular Technology Conference, Rhodes, Greece, 2001.

[15] A. LaMarca, Y. Chawathe, S. Consolvo, J. Hightower, I. Smith, J. Scott, T. Sohn, J. Howard, J. Hughes, F. Potter, J. Tabert, P. Powledge, G. Borriello, B. Schilit, Place lab: Device positioning using radio beacons in the wild, in: Proceedings of the Third International Conference on Pervasive Computing, in: Lecture Notes in Computer Science, Springer-Verlag, 2005.

[16] L. Luxner, The Manhattan Project: AT\&T Wireless invades the Big Apple with microcells, Telephony 8 (20).

[17] N.B. Priyantha, A. Chakraborty, H. Balakrishnan, The cricket location-support system, in: Proceedings of the Sixth Annual ACM International Conference on Mobile Computing and Networking, 2000.

[18] A. Ward, A. Jones, A. Hopper, A new location technique for the active office, IEEE Personal Communications 4 (5) (1997).

[19] M. Youssef, A. Agrawala, U. Shankar, WLAN location determination via clustering and probability distributions, in: Proceedings of the First IEEE Conference on Pervasive Computing and Communications, 2003. 
[20] Ekahau. http://www.ekahau.com.

[21] Ubisense. http://www.ubisense.net.

[22] Versus Technologies. http://www.versustech.com.

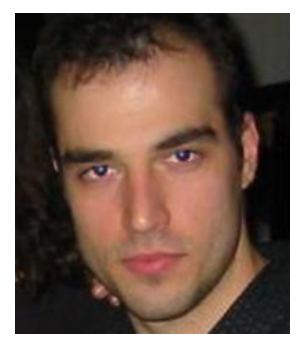

Alex Varshavsky is a Ph.D. student at University of Toronto. His research interests are in mobile and ubiquitous computing. Specifically, his current focus is in localization technologies and and secure pairing of mobile devices. He has a B.Sc. in Computer Science in Computer Science from Technion, Israel and an M.Sc. in Computer Science from the Tel-Aviv University, Israel. He can be contacted at walex@ cs.toronto.edu.

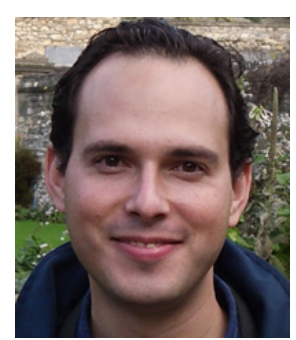

Eyal de Lara is an associate professor in the Department of Computer Science at the University of Toronto. He received a BS degree in computer science from the Instituto Tecnologico y de Estudios Superiores de Monterrey, Mexico, in 1995 and MS and Ph.D. degrees in electrical and computer engineering from Rice University in 1999 and 2002, respectively. His research interests include distributed systems, networking, and mobile and pervasive computing. He is a member of the ACM and the IEEE Computer Society.

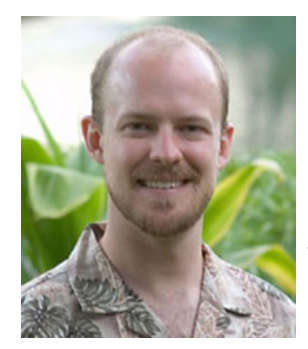

Jeffrey Hightower is a Senior Research Scientist at Intel Research Seattle. His research interests are in devices, services, sensors, and interfaces that help computing calmly fade into the background of daily life. Specifically, his current focus is sensorenhanced mobile computing. He has a BS in Computer Science from the University of Colorado and MS and Ph.D. degrees in Computer Science \& Engineering from the University of Washington. He is a member of the ACM and IEEE. Contact him at jeffrey.r.hightower@intel.com.

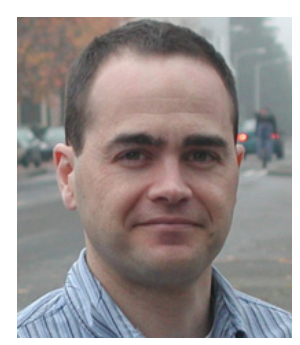

Anthony LaMarca is the associate director of Intel Research Seattle. His research interests include location technologies, ubiquitous computing, distributed systems and human-centered design. He most recently led the Place Lab project which sought to enable wide-scale device positioning using radio beacons. He has a BS in computer science from the University of California at Berkeley and an MS and Ph.D. in computer science from the University of Washington. He can be contacted at anthony.lamarca@intel.com.

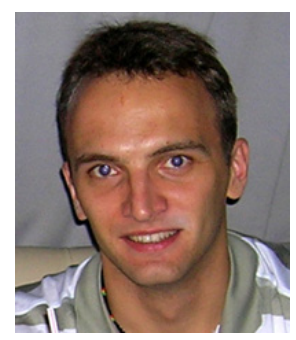

Veljo Otsason is a co-founder and CTO of Mobi Solutions, a mobile software company in Tartu, Estonia. He is interested in data mining, context awareness and mobile payment systems. He received an MS in computer science from the University of Tartu in 2005. 